\title{
Utility of Deep Brain Stimulation Telemedicine for Patients With Movement Disorders During the COVID-19 Outbreak in China
}

\author{
Chencheng Zhang, MD, PhD*11 ${ }^{*}$; Kaiwen Zhu, MD*1 ${ }^{*}$; Zhengyu Lin, MSc* \\ Peng Huang, MD**; Yixin Pan, MD**; Bomin Sun, MD, PhD* ${ }^{*+}$; \\ Dianyou Li, MD, PhD*ث
}

\begin{abstract}
Objective: To explore the utility of deep brain stimulation (DBS) telemedicine in the management of patients with movement disorders from January 2019 to March 2020, covering the main period of the COVID-19 outbreak in China.

Materials and Methods: We obtained data from 40 hospitals around China that employed DBS tele-programming for their outpatients with Parkinson's disease or dystonia from January 2019 to March 2020. Data were obtained on the number and nature of patients' DBS health care service requests, reasons for their requests, the number of DBS telemedicine sessions subsequently completed, safety issues, and the patients' satisfaction with the DBS tele-programing parameter adjustments made.

Results: There were 909 DBS tele-programming health service requests (from 196 patients) completed during the study period. The results showed: 1 ) the number of DBS telemedicine sessions requested and the number of patients examined increased during the COVID-19 outbreak in February and March 2020 when compared with the monthly numbers in 2019; 2) the most common reason for the patients' health service requests was poor symptom control; 3) the most common DBS teleprogramming adjustment made was voltage change; 4) overall, most (89\%) DBS tele-programming adjustment sessions were experienced by the patients as satisfactory; and 5) significant adverse events and unexpected treatment interruptions caused by connection failure or other hardware- or software-related problems did not occur.
\end{abstract}

Conclusions: DBS telemedicine could have a unique role to play in maintaining the delivery of DBS treatment and medical care to outpatients with movement disorders during the COVID-19 pandemic.

Keywords: COVID-19, deep brain stimulation, movement disorders, patient satisfaction, telemedicine

Conflict of Interest: The authors have no conflicts of interest to declare.

\section{INTRODUCTION}

Deep brain stimulation (DBS) is a well-established neurosurgical treatment for movement disorders, such as Parkinson's disease (PD) and dystonia (1). DBS is usually well tolerated and beneficial but personalized postoperative DBS parameter adjustment sessions at the outpatient DBS clinic are essential for achieving optimal therapeutic effects. Unfortunately, frequent face-to-face DBS parameter adjustment sessions put an extra burden on patients' time, effort, and expenses for traveling between clinic and home, especially for patients who live far from medical centers (2). More serious still, the outbreak of COVID-19 and implementation of protective measures, including lockdown of affected cities, quarantines, and instructions to stay at home, have made it extremely challenging, if not impossible, to provide outpatients with personalized DBS parameter adjustments and medical care. Furthermore, it became soon clear that COVID-19 infections are particularly dangerous and often lethal for elderly people who have chronic medical comorbidities (3). This information raises additional clinical concerns because most outpatients treated with DBS for PD are at an advanced age and suffer from multiple comorbidities, making them highly vulnerable to the serious health risk conferred by a COVID-19 infection (4). As a result, the COVID-19 pandemic is catalyzing the application of telemedicine to maintain the delivery of routine health care to patients with chronic neurological diseases (5).

Address correspondence to: Dianyou Li, MD, PhD, Department of Neurosurgery, Ruijin Hospital, Shanghai JiaoTong University School of Medicine, Shanghai, China. Email: lyd11483@rjh.com.cn

\footnotetext{
* Department of Neurosurgery, Ruijin Hospital, Shanghai JiaoTong University School of Medicine, Shanghai, China; and

+ Center for Functional Neurosurgery, Ruijin Hospital, Shanghai JiaoTong University School of Medicine, Shanghai, China
}

${ }^{1}$ These two authors contribute equally to this work.

For more information on author guidelines, an explanation of our peer review process, and conflict of interest informed consent policies, please go to http:// www.wiley.com/WileyCDA/Section/id-301854.html

Source(s) of financial support: No targeted funding for this study.

This is an open access article under the terms of the Creative Commons Attribution-NonCommercial License, which permits use, distribution and reproduction in any medium, provided the original work is properly cited and is not used for commercial purposes. 
DBS telemedicine is an evolving technique that enables clinicians to remotely make therapeutic DBS parameter (e.g., voltage, pulse width, stimulation frequency) adjustments, clinical examinations, battery checks, and technical troubleshooting while outpatients are at home. Furthermore, telemedicine can provide patients with electronic prescribing, medication titration, medical consults, and online psychological support (6-10). However, DBS telemedicine has not yet been incorporated into routine clinical care because this technique is relatively novel and not widely available, requires familiarity and acceptance among primary physicians and patients, and involves specialized medical expertise (6-8). Notwithstanding, telemedicine allows the delivery of DBS treatment and care to patients in their own home environment, without the need for traveling to the outpatient DBS clinic, thereby also minimizing their risk of contracting COVID-19. Thus, the use of telemedicine may not only increase patient satisfaction with DBS treatment under regular societal conditions but may also be crucial to maintain health-care delivery in disasters and public health emergencies, especially now during the COVID-19 pandemic $(9,10)$.

In this study, therefore, we explored the role and value of DBS telemedicine in maintaining the delivery of DBS treatment and medical care to outpatients with movement disorders who had to remain at home during the COVID-19 outbreak in China. Data were obtained from 40 hospitals across the country that used DBS telemedicine to treat patients with movement disorders. We focused on the number and nature of patients' DBS service requests, reasons for their requests, the number of DBS telemedicine sessions subsequently completed, safety issues, and patient satisfaction during the period from January 2019 to March 2020. The lockdown of Wuhan and other protective measures were put into place on January 23, 2020. This megacity in central China started lifting outbound travel restrictions from April 8, 2020, after almost 11 weeks of lockdown due to the COVID-19 outbreak. The study period, thus, covered the period before and during the main period of the COVID-19 outbreak in China.

\section{MATERIALS AND METHODS}

\section{Hospitals and Patients}

Study participants were recruited from 40 hospitals located across China (Supporting Information Table S1) by means of reviewing all the individual medical records of their patients with movement disorders (PD and dystonia) treated with DBS. To control for variations in DBS telemedicine systems used among hospitals, we selected only those hospitals that utilized the same telemedicine system (provided by SceneRay Suzhou, as a free supplemental device to their DBS products). Patients were included only if they: 1) were diagnosed with PD or dystonia and 2) underwent one or more DBS tele-programming sessions at home during the period from January 2019 to March 2020. Ultimately, a total of 196 patients fulfilled these criteria and were included in the study. These patients $(N=196)$ requested and received a total of 909 DBS tele-programming parameter adjustment sessions during the study period. We subdivided the patients' DBS telemedicine sessions ( $N=909$ ) into six groups based on the patient's clinical diagnosis (PD or dystonia) and the specific DBS target used (Table 1). All patients provided informed consent before participating in the study. The study protocol was approved by the institutional research board.

\section{Data Collection}

All hospitals provided data on the number and nature of patients' DBS health-care service requests, reasons for their requests, the number of DBS telemedicine sessions subsequently completed, safety issues, and the patients' satisfaction with the DBS telemedicine treatment and medical care delivered to their homes during the period from January 2019 to March 2020.

\section{DBS Telemedicine System}

The DBS tele-programming system used was a synchronous system, which allowed interactive doctor-patient video connections that transmitted information in both directions at the same time. It consisted of four components (see Supporting Information Fig. S1): 1) the physician client, 2) the patient client, 3) the server station, and 4) the video communication system. The remote programming of the implanted neurostimulator worked via house Wi-Fi and specialized encryption technology was applied to ensure secure and stable data transmission.

1. The physician client consisted of a personal computer (PC) installed with a DBS tele-programming system, which may be simply located at the health professional's office. It provided health-care professionals with a secured login interface, realtime updated patient lists, a tele-programming interface, results display, and a follow-up history page.

2. The patient client consisted of four parts: a digital camera, a television (TV) connected to the digital camera through a highdefinition multimedia interface, an external programmer connected to the digital camera through a Bluetooth dongle, and an in vivo deep brain stimulator communicating with the external programmer through its RF coils. This component provided the patients with a secured login interface, real-time video communications with caregivers, and parameter adjustment information that was transmitted to the stimulator.

3. The server station consisted of three servers: windows communication foundation (WCF) server, website server, and database server. The website server transmitted data between the physician client and the WCF server. The latter provided communication between clients and stored the data in the database server.

4. The video communication system provided real-time high-definition video calls (960p [1280*960], VGA [640*480], frame rate $25 \mathrm{fps}$ ), along with a focus and angle adjustment option to help with symptom assessments.

\section{Implementation of DBS Telemedicine System}

The following procedure was undertaken to implement the telemedicine DBS system in each hospital:

1. The patient was required to schedule an appointment through the website or by phone call.

2. On the appointment day, the patient at his or her home turned on the digital camera and external programmer and connected them to the Internet.

3. The health-care professional logged into the tele-programming software, selected the patient from the appointment list, and started real-time video communication with the patient.

4. If stimulator parameter adjustment was needed, the clinician would establish a connection to the external programmer via a data center, which transmitted the appropriate signals to the impulse generator (IPG). The IPG then delivered feedback on 

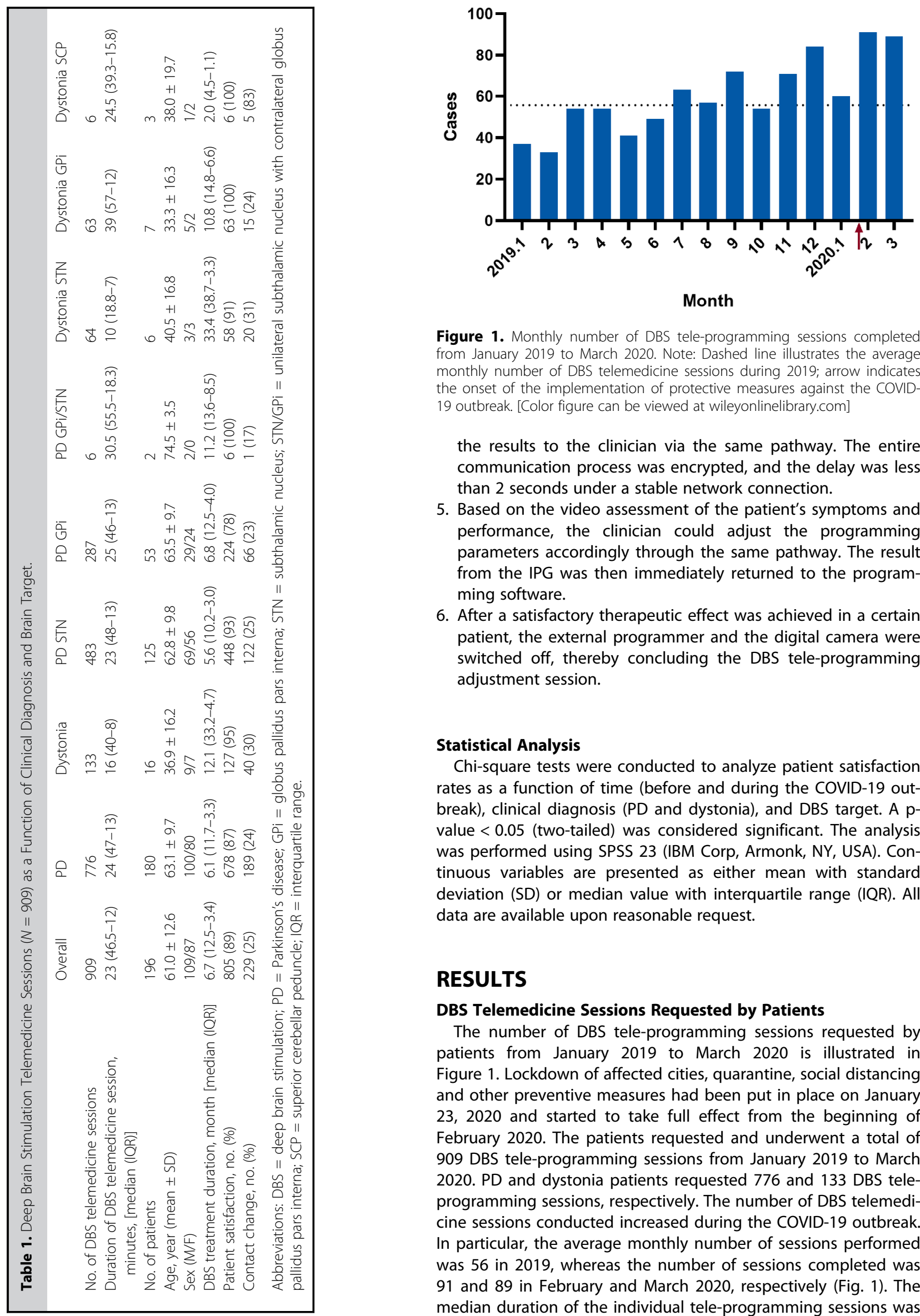

Figure 1. Monthly number of DBS tele-programming sessions completed from January 2019 to March 2020. Note: Dashed line illustrates the average monthly number of DBS telemedicine sessions during 2019; arrow indicates the onset of the implementation of protective measures against the COVID19 outbreak. [Color figure can be viewed at wileyonlinelibrary.com]

the results to the clinician via the same pathway. The entire communication process was encrypted, and the delay was less than 2 seconds under a stable network connection.

5. Based on the video assessment of the patient's symptoms and performance, the clinician could adjust the programming parameters accordingly through the same pathway. The result from the IPG was then immediately returned to the programming software.

6. After a satisfactory therapeutic effect was achieved in a certain patient, the external programmer and the digital camera were switched off, thereby concluding the DBS tele-programming adjustment session.

\section{Statistical Analysis}

Chi-square tests were conducted to analyze patient satisfaction rates as a function of time (before and during the COVID-19 outbreak), clinical diagnosis (PD and dystonia), and DBS target. A pvalue $<0.05$ (two-tailed) was considered significant. The analysis was performed using SPSS 23 (IBM Corp, Armonk, NY, USA). Continuous variables are presented as either mean with standard deviation (SD) or median value with interquartile range (IQR). All data are available upon reasonable request.

\section{RESULTS}

\section{DBS Telemedicine Sessions Requested by Patients}

The number of DBS tele-programming sessions requested by patients from January 2019 to March 2020 is illustrated in Figure 1. Lockdown of affected cities, quarantine, social distancing and other preventive measures had been put in place on January 23, 2020 and started to take full effect from the beginning of February 2020. The patients requested and underwent a total of 909 DBS tele-programming sessions from January 2019 to March 2020. PD and dystonia patients requested 776 and 133 DBS teleprogramming sessions, respectively. The number of DBS telemedicine sessions conducted increased during the COVID-19 outbreak. In particular, the average monthly number of sessions performed was 56 in 2019, whereas the number of sessions completed was 91 and 89 in February and March 2020, respectively (Fig. 1). The median duration of the individual tele-programming sessions was 


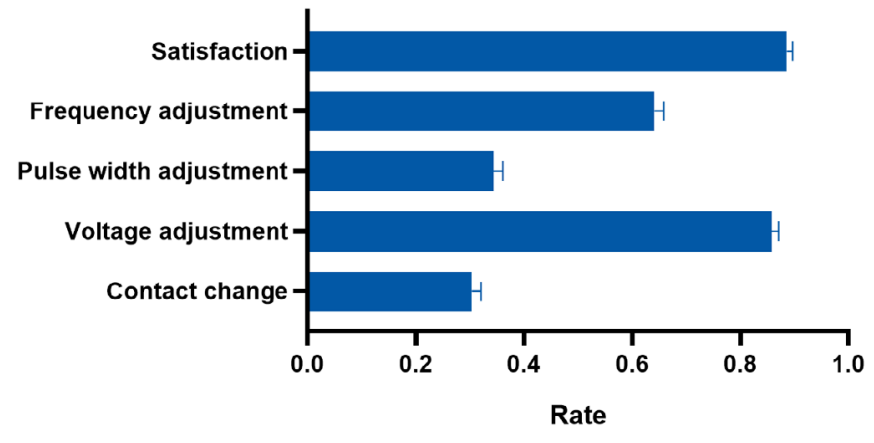

Figure 2. Patient satisfaction and type of DBS tele-programming parameter adjustments (with standard error of the mean). [Color figure can be viewed at wileyonlinelibrary.com]

23.0 minutes (IQR: 46.5-12.0) (Table 1). A table of the mean number of DBS telemedicine sessions, along with DBS and patient characteristics, before the COVID-19 outbreak (in 2019) and during the outbreak (February and March 2020) is given in the Supporting Information Table S2.

\section{Reasons for Requesting DBS Tele-Programming Adjustment Sessions}

The most common reason for requesting DBS tele-programming parameter adjustment sessions $(N=909)$ was poor symptom control ( $n=669$ ) in 2019. Similarly, the most common reason was poor symptom control $(n=170)$ in the first three months of 2020. Notably, in February and March 2020, several patients $(n=10)$ requested their first DBS postoperative parameter adjustment session to be conducted by using DBS tele-processing. In contrast, none of the patients requested DBS tele-processing for the first DBS postoperative adjustment session in 2019.

\section{Adjustments Made to DBS Tele-Programming Parameters}

Therapeutic adjustments made to DBS parameters had been documented for 753 out of the 909 DBS tele-programming sessions. Adjustments were made to voltage level in 646 sessions (85.8\%), pulse width in 259 sessions (34.4\%), stimulation frequency in 483 sessions (64.1\%), and contact site in 229 sessions (25.2\%) (Fig. 2).

\section{Safety Profile of DBS Tele-Programming}

No severe adverse effects other than stimulation-related reversible side effects were reported. There were no unstable network connections during the DBS tele-programming sessions or any hardwarerelated problems that intervened or interrupted the DBS treatment and medical care delivered to the patients at their homes.

\section{Patient Satisfaction}

Overall, across patients' diagnoses and DBS targets, 805 out of 909 DBS tele-programming parameter adjustment sessions (89\%) were experienced by the patients as satisfactory and clinically useful, whereas 104 DBS telemedicine sessions (11\%) were viewed by patients as having a less favorable outcome than they expected (Fig. 2 and Table 1). In 2019, the percentage of patients who reported satisfaction after DBS tele-programming was significantly lower (87\%, 581 out of 669 patients) than the percentage of patients who expressed satisfaction after the telemedicine session in February and March 2020 (94\%, 169 out of 180 patients) ( $p=0.0090$ ). Patient satisfaction differed as a function of clinical diagnosis, that is, the percentage of patients with PD (87\%, 678 out of 776 patients) who reported satisfaction with the DBS telemedicine session was significantly lower than the satisfaction rate of patients with dystonia $(95 \%, 127$ out of 133 patients) ( $p=0.0066$ ) (Table 1). Satisfaction rates among patients with PD differed as a function of DBS target, namely the satisfaction rate of PD patients treated with STN DBS (93\%, 448 out of 483 patients) was significantly higher than that of PD patients treated with GPi DBS (78\%, 224 out of 278 patients) $(p<0.0001)$ (Table 1). The most commonly performed DBS tele-programming parameter adjustment was a voltage increase, followed by a pulse width increase and a decrease in stimulation frequency (Fig. 2). Contact change was also frequently needed and performed (Table 1).

\section{DISCUSSION}

The main objective of this study was to explore the utility of DBS telemedicine in the daily management of outpatients with movement disorders before and during the COVID-19 outbreak when protective measures were put into place in China. The results showed that 1) the number of DBS tele-programming sessions requested and the number of patients examined increased during the COVID-19 outbreak compared with the corresponding numbers in 2019; 2) the most common reason for DBS tele-programming requests was poor symptom control; 3) the most common DBS tele-programming adjustment made was voltage change, followed by adjustments of pulse width and stimulation frequency; contact changes were also frequently performed; 4) overall, most (89\%) DBS tele-programming adjustment sessions were experienced by the patients as satisfactory, but satisfaction rates were higher among dystonia patients (95\%) than PD patients (87\%) and higher among PD patients treated with STN DBS (93\%) than PD patients treated with GPi DBS (78\%); and 5) DBS telemedicine was safe: significant adverse events and unexpected treatment interruptions by connection failure or other hardware- or software-related problems did not occur. These results provide tentative evidence supporting the feasibility, safety, and clinical utility of DBS telemedicine in general and, specifically, its crucial role in maintaining the delivery of DBS treatment and medical care to outpatients with movement disorders who need to remain at home during the COVID-19 pandemic.

Overall, patient satisfaction rates after the DBS telemedicine parameter adjustment sessions were high, in line with the results from previous DBS telemedicine studies $(11,12)$ This is an important finding because patient satisfaction is commonly used as an indicator of the quality of health care, although the overall level of satisfaction reported by patients may also be related to their expectations (13). Indeed, there were 52 telemedicine sessions in which no DBS tele-programming parameter adjustments or contact changes were performed, yet the patients reported to be satisfied with many of these sessions, too. This observation suggests that the patients' reported levels of satisfaction were related not only to the DBS tele-processing adjustment itself, but also to their expectations and the quality of the psychological support and medical consults they received during the sessions.

We also observed that the monthly number of DBS telemedicine sessions requested by patients was higher during the COVID-19 outbreak in February and March 2020 than the monthly number of telemedicine sessions requested in 2019. There was also an increase in the number of patients who requested DBS telemedicine in February and March 2020, along with higher patient satisfaction rates. Additionally, a considerable number of patients requested their first postoperative parameter adjustments through DBS tele-programming, 
which did not happen in 2019. Although these results indicate that there was a greater need for DBS telemedicine during the COVID-19 outbreak, the possibility exists that patients and primary physicians had become more familiar with DBS telemedicine at that time (8). Indeed, it seems that the number of telemedicine sessions and patients examined gradually increased already from the beginning of 2019, without showing abrupt, high peaks during the COVID-19 outbreak in February and March 2020. Thus, the observed increase in the number of DBS telemedicine sessions and patients in February and March 2020 could reflect both an increased familiarity and acceptance of this novel technique and a greater need due to the COVID-19 outbreak and implementation of protective measures.

Various telemedicine techniques have long been available in many countries, but they have played, so far, only a minor role in the delivery of routine health care $(5,14-16)$. In China, DBS telemedicine techniques have been available for several years, but their use has similarly not yet been incorporated into routine clinical practice. The COVID-19 pandemic may modify this situation. DBS tele-programming turned out to be an essential tool for maintaining the delivery of DBS treatment and medical care to patients at their own homes, which was otherwise not possible during this unprecedented health crisis. As a consequence, the COVID-19 pandemic is expected to trigger substantial research efforts and resources to develop DBS telemedicine techniques further and to integrate them into the healthcare system.

This study has several limitations that need to be acknowledged. First, we did not make a comparison between the clinical effectiveness of DBS telemedicine and routine face-to-face DBS treatment using standardized clinical rating scales. This remains an important topic for further research. Second, we selected hospitals that all used the same DBS telemedicine system. It remains uncertain, therefore, whether the results can be generalized to hospitals using other DBS telemedicine system. In this context, it should be noted that some telemedicine systems utilize a smart phone or iPad instead of a specialized system. It would be interesting to compare patient convenience, cost-effectiveness, and safety between different DBS telemedicine platforms in future studies. Third, the study period covered the main, and not the entire, period of the COVID-19 outbreak in China. We intend to report data obtained before, during, and after the COVID-19 outbreak in a future publication, which will provide a more comprehensive picture of the role and value of DBS telemedicine for outpatients with movement disorders in public health emergencies.

\section{Authorship Statements}

Dr. Chencheng Zhang designed and conceptualized study and drafted the manuscript, providing intellectual content. Dr. Kaiwen Zhu was responsible for the acquisition of data, analysis of the data, drafting the manuscript, providing intellectual content. Dr. Zhengyu Lin analyzed and interpreted the data and revised the manuscript for intellectual content. Dr. Peng Huang was responsible for the acquisition of data and revised the manuscript for intellectual content. Dr. Yixin Pan was responsible for the acquisition of data and revised the manuscript for intellectual content. Dr. Dianyou Li designed and conceptualized the study, interpreted the data and revised the manuscript for intellectual content. Dr. Bomin Sun interpreted the data and revised the manuscript for intellectual content. All authors approved the final manuscript.

\section{How to Cite this Article:}

Zhang C., Zhu K., Lin Z., Huang P., Pan Y., Sun B., Li D. 2021.
Utility of Deep Brain Stimulation Telemedicine for Patients With Movement Disorders During the COVID-19 Outbreak in China.

Neuromodulation 2021; 24: 337-342

\section{REFERENCES}

1. Okun MS. Deep-brain stimulation - Entering the era of human neural-network modulation. N Engl J Med 2014;371:1369-1373. https://doi.org/10.1056/ NEJMp1408779.

2. Deuschl G, Herzog J, Kleiner-Fisman G et al. Deep brain stimulation: postoperative issues. Mov Disord 2006;21:S219-S237. https://doi.org/10.1002/mds. 20957.

3. Wu Z, McGoogan JM. Characteristics of and important lessons from the coronavirus disease 2019 (COVID-19) outbreak in China: summary of a report of 72314 cases from the Chinese Center for Disease Control and Prevention. JAMA 2020 323:1239. https://doi.org/10.1001/jama.2020.2648.

4. Antonini A, Leta V, Teo J, Chaudhuri KR. Outcome of Parkinson's disease patients affected by COVID-19. Mov Disord 2020;29:905-908. https://doi.org/10.1002/mds. 28104.

5. Bloem BR, Dorsey ER, Okun MS. The coronavirus disease 2019 crisis as catalyst for telemedicine for chronic neurological disorders. JAMA Neurol 2020;24:927. https://doi.org/10.1001/jamaneurol.2020.1452.

6. Zhang C, Li D, Zeljic K, Tan H, Ning Y, Sun B. A remote and wireless deep brain stimulation programming system. Neuromodulation 2016;19:437-439. https://doi. org/10.1111/ner.12448.

7. Zhang $C$, Zhang $Y$, Zhan $S$ et al. Telemedical deep brain stimulation: merits and limitations. Stereotact Funct Neurosurg 2018;96:272-273. https://doi.org/10.1159/ 000491603.

8. Chirra M, Marsili L, Wattley L et al. Telemedicine in neurological disorders: opportunities and challenges. Telemed J E Health 2019;25:541-550. https://doi.org/10. 1089/tmj.2018.0101.

9. Hollander JE, Carr BG. Virtually perfect? Telemedicine for Covid-19. N Engl J Med 2020;382:1679-1681. https://doi.org/10.1056/NEJMp2003539.

10. Fasano A, Antonini A, Katzenschlager $R$ et al. Management of advanced therapies in Parkinson's disease patients in times of humanitarian crisis: the COVID-19 experience. Mov Disord Clin Pract 2020;7:361-372. https://doi.org/ $10.1002 / \mathrm{mdc} 3.12965$.

11. Jitkritsadakul O, Rajalingam R, Toenjes C, Munhoz RP, Fasano A. Tele-health for patients with deep brain stimulation: the experience of the Ontario telemedicine network: tele-health for patients with DBS. Mov Disord 2018;33:491-492. https:// doi.org/10.1002/mds.27230.

12. Chen $\mathrm{Y}, \mathrm{Hao} \mathrm{H}$, Chen $\mathrm{H}, \mathrm{Li}$ L. The study on a telemedicine interaction mode for deep brain stimulation postoperative follow-up. Conf Proc IEEE Eng Med Biol Soc 2015;186-189. https://doi.org/10.1109/EMBC.2015.7318331.

13. Bjertnaes $\mathrm{OA}$, Sjetne $\mathrm{IS}$, Iversen $\mathrm{HH}$. Overall patient satisfaction with hospitals: effects of patient-reported experiences and fulfilment of expectations. BMJ Qual Saf 2012;21:39-46. https://doi.org/10.1136/bmjqs-2011-000137.

14. Srinivasan R, Ben-Pazi $H$, Dekker $M$ et al. Telemedicine for hyperkinetic movement disorders. Tremor Other Hyperkinet Mov 2020;10. https://doi.org/10.5334/ tohm.534.

15. Klein BC, Busis NA. COVID-19 is catalyzing the adoption of teleneurology. Neurology 2020;94:903-904. https://doi.org/10.1212/WNL.0000000000009494.

16. Smith $A C$, Thomas $E$, Snoswell $C L$ et al. Telehealth for global emergencies: implications for coronavirus disease 2019 (COVID-19). J Telemed Telecare 2020;26: 309-313. https://doi.org/10.1177/1357633X20916567.

\section{SUPPORTING INFORMATION}

Additional supporting information may be found online in the supporting information tab for this article.

\section{COMMENT}

The authors present a straightforward study delineating their use of a remote telemedicine platform for programming DBS devices. They report an increase in the use of this technology during the COVID-19 pandemic in China and high patient satisfaction. The COVID-19 pandemic has forced all of us to modify our practice of 
medicine and it is clear that telemedicine is an adaptation that will persist. In retrospect it seems absurd that it took this catastrophic event for CMS and private US insurers to reimburse telemedicine visits, instead insisting that the elderly and infirmed be transported to a clinic, at great expense and effort, for many matters that could easily be addressed via telemedicine without a decrease in the quality of care provided. The widespread use of these sorts of remote programming systems is long overdue.

Ronald Alterman, MD Boston, MA USA 